\title{
EXISTENCE AND UNIQUENESS OF ALGEBRAIC CURVATURE TENSORS WITH PRESCRIBED PROPERTIES AND AN APPLICATION TO THE SPHERE THEOREM
}

\author{
WALTER SEAMAN
}

\begin{abstract}
An existence and uniqueness theorem is proved for algebraic curvature tensors and then applied to yield a global geometric theorem for locally weakly quarter pinched Riemannian manifolds whose second Betti number is nonzero.
\end{abstract}

\section{INTRODUCTION}

The Sphere Theorem (cf. [3]) states

(1.1) If $M$ is a complete, simply-connected Riemannian manifold whose sectional curvature satisfies $4 \geq K>1$, then $M$ is homeomorphic to a sphere. If $4 \geq K \geq 1$ then $M$ is either homeomorphic to a sphere or is isometric to a compact symmetric space.

Note that in the latter case, $M$ is necessarily a rank one symmetric space, as it is positively curved.

In $[4,5]$, the Sphere Theorem has been generalized as follows:

(1.2) If $M$ is a complete Riemannian manifold with $K \geq 1$ and $\operatorname{diam}(M)>$ $\pi / 2$, then $M$ is homeomorphic to a sphere. If $K \geq 1$ and $\operatorname{diam}(M)=\pi / 2$, then $M$ is either homeomorphic to a sphere, or the universal cover of $M, \widetilde{M}$, is isometric to a compact (rank one) symmetric space except possibly when $M$ has the integral cohomology ring of the Cayley plane $\mathbb{C a} P^{2}$. See [4] also for results in the nonsimply-connected case.

From (1.1), or in all but one case of (1.2), one concludes, by means of the classification of symmetric spaces [6], or by the work in [4] on Riemannian submersions, that the only possibilities for $\widetilde{M}$ are: $\widetilde{M}$ is topologically a sphere or is isometric to a projective space over the complex, quaternionic or Cayley numbers (and of course each of these possibilities do have the requisite properties).

(1.3) Theorem. Let $M$ be a compact orientable Riemannian manifold, whose second Betti number, $b_{2}$, is nonzero. Suppose there is a function $\Delta: M \rightarrow[0, \infty)$ such that for all $p \in M, \Delta(p) \geq K(p) \geq \Delta(p) / 4$, where $K(p)$ is the sectional

Received by the editors November 11, 1988. The results of this paper were presented at the Geometry and Topology Conference at Lehigh University, May 1988.

1980 Mathematics Subject Classification (1985 Revision). Primary 53C20. 
curvature (function) of $M$ at $p$. Then $\Delta$ is a constant function: if $M$ is odd-dimensional, then $\Delta \equiv 0$, so $M$ is flat; if $\operatorname{dim} M=2 n$ then either $\Delta \equiv 0$, so $M$ is again flat, or $\Delta$ is a positive constant, and then $M$ can be made into a Kähler manifold with constant holomorphic sectional curvature $\Delta$, and so (since $M$ is simply-connected by Synge's theorem) $M$ is biholomorphically isometric to $\mathbb{C} P^{n}$, the complex projective space with metric $\frac{1}{\Delta} g_{\text {can }} \quad(c f . \S 3)$.

If one starts with the assumption that $\Delta$ is a positive constant function, then the above theorem, with compactness replaced by completeness, can be deduced, for example, by the Sphere Theorem and the classification of symmetric spaces by the process of elimination. Our proof of Theorem 1.3 does not make use of the classification of symmetric spaces, but shows "directly" that if $M$ is evendimensional, at each $p \in M$, the curvature tensor $R_{p}$ satisfies $R_{p}=\Delta(p)$. $R_{\mathbb{C} P^{n}}$, where $R_{\mathbb{C} P^{n}}$ is the algebraic curvature tensor for $\mathbb{C} P^{n}$, with sectional curvature pinched between 1 and $1 / 4$. If $M$ is not flat, the assumptions on the sectional curvature and $b_{2} \neq 0$ yield the existence on $M$ of a parallel orthogonal almost complex structure, from which $M$ gets its Kähler structure. The conclusion then follows from the rigidity of constant holomorphic sectional curvature Kähler manifolds [9, p. 170], and hence is considerably simpler than using the classification of symmetric spaces together with the Sphere Theorem.

In order to prove Theorem 1.3, we prove an existence and uniqueness theorem for algebraic curvature tensors satisfying certain prescribed properties. This can be explained as follows: let $R$ be an algebraic curvature tensor on an even-dimensional vector space $V^{2 n}$ with positive definite inner product $\langle$,$\rangle .$ Suppose the sectional curvature of $R, K$, satisfies $\Delta \geq K \geq \delta$. It is known (and we give a short proof in $\S 2)$ that the Weitzenböck operator $R_{2}=\operatorname{Ric} \bigwedge\langle\rangle-$, $2 \widehat{R}$ (see $\S 2$ for notation) has minimum possible eigenvalue $(n-1) \frac{4}{3}(4 \delta-\Delta)$. This idea was originally used in [1] to show that a compact even-dimensional manifold with $1 \geq K>\frac{1}{4}$ has $b_{2}=0$. This result is of course sharp, and furthermore the Weitzenböck operator for $R_{\mathbb{C} P^{n}}$ (with sectional curvature $1 \geq$ $\left.K \geq \frac{1}{4}\right)$ has minimum eigenvalue $0\left(=(n-1) \frac{4}{3}\left(4 \cdot \frac{1}{4}-1\right)\right)$ with eigenvector " $J$ ", the complex structure on $V$ used to define $R_{\mathbb{C} P^{n}}$. In [1] there is also a condition guaranteeing that $b_{2}=0$ in the odd-dimensional case (cf. Proposition 2.9) but it is not known if this result is sharp.

A natural question to ask, strictly from the point of view of algebraic curvature tensors, is the following: given any $\Delta, \delta \in \mathbb{R}, \Delta>\delta$, can one find an algebraic curvature tensor on $V^{2 n}$ such that $\Delta \geq K(R) \geq \delta$, and whose Weitzenböck operator $R_{2}$ has (minimum possible) eigenvalue $(n-1) \frac{4}{3}(4 \delta-\Delta)$ ? If so, how "many" such algebraic curvature tensors are there? The answer to these questions is contained in the following theorem.

(1.4) Theorem. (i) (Existence) Let $J$ be an orthogonal complex structure on $V^{2 n}$ and define $\widetilde{R}:=\frac{4}{3}(\Delta-\delta) R_{\mathbb{C}^{n}}+\frac{1}{3}(4 \delta-\Delta)$ Id. Then $\widetilde{R}$ is an algebraic curvature tensor whose sectional curvature, $\widetilde{K}$, satisfies $\Delta \geq \widetilde{K} \geq \delta, \widetilde{K}=\Delta$ 
on a 2-plane, $P \Leftrightarrow P$ is $J$-invariant, $\widetilde{K}=\delta \Leftrightarrow P$ is anti-J invariant, and the Weitzenböck operator for $\widetilde{R}, \widetilde{R}_{2}$, has (minimum) eigenvalue $(n-1) \frac{4}{3}(4 \delta-\Delta)$.

(ii) (Uniqueness) If $R$ is an algebraic curvature tensor on $V^{2 n}$ whose sectional curvature $K$ satisfies $\Delta \geq K \geq \delta$, and whose Weitzenböck operator $R_{2}$ has (minimum) eigenvalue $(n-1) \frac{4}{3}(4 \delta-\Delta)$, then there is an orthogonal complex structure $J$ on $V$ such that $R=\widetilde{R}$.

Corollary. $\Delta \cdot R_{\mathbb{C} P^{n}}, \Delta \geq 0, n \geq 2$, can be characterized as the unique algebraic curvature tensor on an even-dimensional vector space whose sectional curvature satisfies $\Delta \geq K \geq \frac{\Delta}{4}$ and whose Weitzenböck operator has zero as an eigenvalue.

Once Theorem 1.4 has been established, Theorem 1.3 follows quickly (cf. $\S 3)$.

Theorem 1.4 is also valid in odd dimensions, in the following sense: if $\Delta \geq$ $K \geq \delta$ on $V^{2 n+1}$, then $R_{2} \geq \frac{2}{3}((8 n-5) \delta-2 \Delta(n-1)$ ) (cf. Proposition 2.9). If one has equality, then there is an orthogonal complex structure $J$ on a $2 n$ dimensional subspace, $W$, of $V$, and $\left.J\right|_{W^{\perp}}=0$, such that $R=\widetilde{R}$. This can be deduced from the even-dimensional case and the proof of Theorem 1.4. Since the odd-dimensional portion of Theorem 1.3 does not use this uniqueness property, but only the lower bound for $R_{2}$ given above, we omit the proof.

After the initial writing of this paper, $[\mathrm{H}]$ was brought to our attention, and the author of that paper informed us that the above Corollary can be deduced from Lemmas 6, 7, and 8 of $[\mathrm{H}]$.

\section{BACKGROUND, NOTATION, AND LINEAR ALGEBRA}

Let $V$ be a real vector space with positive definite inner product $\langle$,$\rangle .$ Using $\langle$,$\rangle , we identify V$ with $V^{*}$ and $\wedge V$ with $\wedge V^{*}$. The inner product on $V$ is extended to $\Lambda^{2} V$ by bilinearity and the prescription: $\langle a \wedge b$, $c \wedge d\rangle=\langle a, c\rangle\langle b, d\rangle-\langle a, d\rangle\langle b, c\rangle$, where $a, b, c, d \in V$. For $X \in \Lambda^{2} V$, we define the associated skew symmetric operator on $V$, again called $X$, by $\langle X(a), b\rangle:=\langle X, b \wedge a\rangle$ (so, in particular, if $X=x \wedge y, x, y \in V$, then $x \wedge y(a)=\langle y, a\rangle x-\langle x, a\rangle y)$.

An algebraic curvature tensor on $V$ may be defined as a multilinear map $R: V \times V \times V \rightarrow V$ satisfying

$$
\begin{gathered}
\langle R(x, y) z, w\rangle=\langle R(z, w) x, y\rangle, \\
\langle R(x, y) z, w\rangle=-\langle R(y, x) z, w\rangle, \\
R(x, y) z=R(x, z) y+R(z, y) x \quad \text { (the Bianchi identity), }
\end{gathered}
$$

$x, y, z, w \in V$. If $P$ is a two-dimensional plane in $V$, with orthonormal basis $v_{1}, v_{2}$, the sectional curvature of $P$ is (invariantly) defined to be $K(P)=$ $\left\langle R\left(v_{1}, v_{2}\right) v_{2}, v_{1}\right\rangle$. As is well known, $K$ determines $R$. The (algebraic) curvature operator, $\widehat{R}$, is the symmetric linear endomorphism of $\wedge^{2} V$ defined by $\langle\widehat{R}(x \wedge y), w \wedge z\rangle=\langle R(x, y) z, w\rangle$. The Ricci tensor is the symmetric bilinear form Ric: $V \times V \rightarrow \mathbb{R}$ (invariantly) defined by $\operatorname{Ric}(x, y)=\sum_{i}\left\langle R\left(e_{i}, x\right) y, e_{i}\right\rangle$ 
where $\left\{e_{i}\right\}$ is any orthonormal basis of $V$. (On a Riemannian manifold with metric $\langle$,$\rangle and connection \nabla$, we have $R(X, Y) Z=\nabla_{X} \nabla_{Y} Z-\nabla_{Y} \nabla_{X} Z-$ $\nabla_{[X Y]} Z$, for $X, Y, Z$ vector fields, yielding an algebraic curvature tensor at each point.) The Weitzenböck operator of $R$ (on $V$ ) is the symmetric linear transformation, $R_{2}: \Lambda^{2} V \rightarrow \Lambda^{2} V$, defined by $R_{2}=\operatorname{Ric} \Lambda\langle\rangle-,2 \widehat{R}$, i.e., for $v_{1}, v_{2}, w_{1}, w_{2} \in V$,

$$
\begin{aligned}
\left\langle R_{2}\left(v_{1} \wedge v_{2}\right), w_{1} \wedge w_{2}\right\rangle= & \operatorname{Ric}\left(v_{1}, w_{1}\right)\left\langle v_{2}, w_{2}\right\rangle+\operatorname{Ric}\left(v_{2}, w_{2}\right)\left\langle v_{1}, w_{1}\right\rangle \\
& -\operatorname{Ric}\left(v_{1}, w_{2}\right)\left\langle v_{2}, w_{1}\right\rangle-\operatorname{Ric}\left(v_{2}, w_{1}\right)\left\langle v_{1}, w_{2}\right\rangle \\
& -2\left\langle R\left(v_{1}, v_{2}\right) w_{2}, w_{1}\right\rangle .
\end{aligned}
$$

$R_{2}$ is important since it is the 0th order term appearing in the Weitzenböck formula for the Laplacian acting on 2-forms on a Riemannian manifold $M$ (cf. [11, Chapter 4]):

$$
\Delta X=-\operatorname{div} \nabla X+R_{2} X .
$$

For any $X \in \Lambda^{2}(T M),(2.5)$ yields

$$
\langle\Delta X, X\rangle=\frac{1}{2} \Delta|X|^{2}+|\nabla X|^{2}+\left\langle R_{2} X, X\right\rangle .
$$

On an oriented compact $M$, integrating (2.6) (the well-known Bochner technique) shows that if $R_{2}$ is a pointwise nonnegative operator, then every harmonic 2-form $X \quad(\Delta X \equiv 0)$ is parallel $(\nabla X \equiv 0)$ and hence $R_{2} X \equiv 0$ (from (2.5)). If $R_{2}$ is positive definite, then there can be no harmonic two-forms, hence $b_{2}(M)=0$ from the Hodge-DeRham Theorem. It is therefore of interest to know which pointwise assumptions on the sectional curvature guaranteed that $R_{2}$ is (pointwise) a nonnegative or positive definite operator. This question is resolved in [1] and we present a proof suited to our goals. Our proof is based on estimates for the components of an algebraic curvature tensor, which are sharp for $R_{\mathbb{C} P^{n}}$. Our notation is as follows: for $\left\{e_{i}\right\}$ an orthonormal basis of $V, R_{i j k l}:=\left\langle R\left(e_{i}, e_{j}\right) e_{k}, e_{l}\right\rangle$ (sometimes denoted $\left.R_{i, j, k, l}\right)$; the sectional curvature of the plane spanned by $e_{i}, e_{j}$ is denoted $K_{i j}$; the sectional curvature of the plane spanned by $\left(e_{i} \pm e_{j}\right) / \sqrt{2},\left(e_{k} \pm e_{l}\right) / \sqrt{2}$, for $i, j, k, l$ distinct, is denoted $K_{i \pm j, k \pm l}$; and " $K$ " denotes all possible sectional curvatures of $V$.

Proposition 2.7 [7]. If $\Delta \geq K \geq \delta$ and $i, j, k, l$ are distinct, then $\left|R_{i j k l}\right| \leq$ $\frac{2}{3}(\Delta-\delta)$. Furthermore, $R_{i j k l}=\frac{2}{3}(\Delta-\delta)\left(-\frac{2}{3}(\Delta-\delta)\right)$ iff $K_{i+l, j+k}=K_{i-l, j-k}=$ $K_{j-l, i+k}=K_{j+l, i-k}=\Delta(\delta)$ and $K_{i-l, j+k}=K_{i+l, j-k}=K_{j+l, i+k}=K_{j-l, i-k}=$ $\delta(\Delta)$.

Proof. $R_{i j k l}=$

$$
\begin{aligned}
& \frac{1}{6}\left\{K_{i+l, j+k}+K_{i-l, j-k}-K_{i-l, j+k}-K_{i+l, j-k}\right. \\
& \left.\quad+K_{j-l, i+k}+K_{j+l, i-k}-K_{j+l, i+k}-K_{j-l, i-k}\right\} \text {. Q.E.D. }
\end{aligned}
$$

We have written this expression in this way as we will make use of $(2.8 \mathrm{~A})$ and (2.8B) later. We will now always assume $n \geq 2$. 
Proposition 2.9. If $V$ is $2 n$-dimensional, with algebraic curvature tensor $R$ whose sectional curvature satisfies $\Delta \geq K \geq \delta(\Delta>\delta$ being the nontrivial case), then the minimum eigenvalue $r$ of the Weitzenböck operator $R_{2}$ satisfies $r \geq(n-1) \frac{4}{3}(4 \delta-\Delta)$. If $V$ is $(2 n+1)$-dimensional, $r \geq \frac{2}{3}((8 n-5) \delta-2 \Delta(n-1))$. Proof. $R_{2}: \Lambda^{2} V \rightarrow \Lambda^{2} V$ is symmetric by its definition, hence has all real eigenvalues. Let $r=$ the minimum eigenvalue of $R_{2}$ with (nonzero) eigenvector $X$. Then there is an orthonormal basis $\left\{e_{i}\right\}$ of $V$ such that $X=\sum_{i=1}^{n} \lambda_{i} e_{2 i-1} \wedge$ $e_{2 i}$, with $\lambda_{1} \geq \lambda_{2} \geq \cdots \geq \lambda_{n} \geq 0$.

Now compute

$$
\begin{aligned}
r \lambda_{1} & =\left\langle r X, e_{1} \wedge e_{2}\right\rangle=\left\langle R_{2} X, e_{1} \wedge e_{2}\right\rangle=\sum_{i=1}^{n} \lambda_{i}\left\langle R_{2}\left(e_{2 i-1} \wedge e_{2 i}\right), e_{1} \wedge e_{2}\right\rangle \\
& =\lambda_{1}\left\langle R_{2}\left(e_{1} \wedge e_{2}\right), e_{1} \wedge e_{2}\right\rangle+\sum_{i \geq 2} \lambda_{i}\left\langle R_{2}\left(e_{2 i-1} \wedge e_{2 i}\right), e_{1} \wedge e_{2}\right\rangle .
\end{aligned}
$$

From (2.4), we compute

$$
r \lambda_{1}=\lambda_{1} \sum_{j \neq 1,2}\left(K_{j 1}+K_{j 2}\right)-2 \sum_{i \geq 2} \lambda_{i} R_{2 i-1,2 i, 2,1},
$$

where $j$ runs from 3 to $2 n$ if $\operatorname{dim} V=2 n$. Using $\lambda_{1}>0, \lambda_{i} / \lambda_{1} \leq 1$ for all $i, \Delta \geq K \geq \delta$, and Proposition 2.7, (2.11) yields

$$
\begin{aligned}
r & =\sum_{j \neq 1,2}\left(K_{j 1}+K_{j 2}\right)-2 \sum_{i \geq 2} \frac{\lambda_{i}}{\lambda_{1}} R_{2 i-1,2 i, 2,1} \\
& \geq 2 \delta(2 n-2)-2 \sum_{i \geq 2} \frac{\lambda_{i}}{\lambda_{1}}\left|R_{2 i-1,2 i, 2,1}\right| \\
& \geq 4 \delta(n-1)-2 \sum_{i \geq 2}\left|R_{2 i-1,2 i, 2,1}\right| \\
& \geq 4 \delta(n-1)-2 \sum_{i \geq 2} \frac{2}{3}(\Delta-\delta) \\
& =4 \delta(n-1)-\frac{4}{3}(\Delta-\delta)(n-1)=(n-1) \frac{4}{3}(4 \delta-\Delta) .
\end{aligned}
$$

The odd-dimensional case follows in the same way, but now the first sum in $(2.12)$ is $\geq(2 n-1) \cdot 2 \delta$. Q.E.D.

Remark. Notice that if $r=(n-1) \frac{4}{3}(4 \delta-\Delta)$, then the inequalities (2.12a-c) must all be equalities. In particular, if $\operatorname{dim} V=2 n$ and $r=(n-1) \frac{4}{3}(4 \delta-\Delta)$, then we must have $\left|R_{2 i-1,2 i, 2,1}\right|=\frac{2}{3}(\Delta-\delta) \forall_{i} \geq 2((2.12 \mathrm{~b})=(2.12 \mathrm{c}))$ and, since $\Delta-\delta>0$ by assumption, $\lambda_{i}=\lambda_{1} \forall_{i}((2.12 \mathrm{c})=(2.21 \mathrm{~b})=(2.12 \mathrm{a}))$. This yields the following corollary.

Corollary 2.13. With the assumptions of Proposition 2.9, and $\operatorname{dim} V=2 n$ if $r=(n-1) \frac{4}{3}(4 \delta-\Delta)$, then for all $i \neq j$ we have

$$
K_{i j}=\delta \text { except possibly when } i=2 k, j=2 k-1 \text {, }
$$

for some $k$ (or $i, j$ reversed here), 


$$
R_{2 i-1,2 i, 2 j, 2 j-1}=\frac{2}{3}(\Delta-\delta), \quad i \neq j \in\{1, \ldots, n\} .
$$

Proof. As noted above, if $r=(n-1) \frac{4}{3}(4 \delta-\Delta)$, then we may normalize $X$ so that $X=\sum_{i=1}^{n} e_{2 i-1} \wedge e_{2 i}$. Now compute $\forall_{j}$ :

$$
\begin{aligned}
r= & (n-1) \frac{4}{3}(4 \delta-\Delta)=\left\langle r X, e_{2 j-1} \wedge e_{2 j}\right\rangle=\left\langle R_{2} X, e_{2 j-1} \wedge e_{2 j}\right\rangle \\
= & \sum_{i=1}^{n}\left\langle R_{2}\left(e_{2 i-1} \wedge e_{2 i}\right), e_{2 j-1} \wedge e_{2 j}\right\rangle=\left\langle R_{2}\left(e_{2 j-1} \wedge e_{2 j}\right), e_{2 j-1} \wedge e_{2 j}\right\rangle \\
& +\sum_{i \neq j}\left\langle R_{2}\left(e_{2 i-1} \wedge e_{2 i}\right), e_{2 j-1} \wedge e_{2 j}\right\rangle \\
& +\sum_{\substack{l \neq 2 j \\
l \neq 2 j-1 \\
1 \leq l \leq 2 n}}\left(K_{l, 2 j-1}+K_{l, 2 j}\right)-2 \sum_{\substack{i \neq j \\
1 \leq i \leq n}} R_{2 i-1,2 i, 2 j, 2 j-1}
\end{aligned}
$$

(2.16a) $\quad \geq 4 \delta(n-1)-2 \sum_{i \neq j} R_{2 i-1,2 i, 2 j, 2 j-1}$

$(2.16 \mathrm{~b}) \quad \geq 4 \delta(n-1)-\frac{4}{3}(\Delta-\delta)(n-1)$

$$
=(n-1) \frac{4}{3}(4 \delta-\Delta) \text {. }
$$

Thus the inequalities in $(2.16 \mathrm{a})$ and $(2.16 \mathrm{~b})$ must be equalities, which can occur iff $K_{l, 2 j}=K_{l, 2 j-1}=\delta \forall_{j}$ and for any $l \neq 2 j, 2 j-1$, which yields (2.14), and $R_{2 i-1,2 i, 2 j, 2 j-1}=\frac{2}{3}(\Delta-\delta)$ which is (2.15). Q.E.D.

We now pause to show that there actually exists a curvature tensor satisfying the hypotheses of Proposition 2.9 and Corollary 2.13.

Proposition 2.17. Let $\Delta>\delta$ be numbers, $V^{2 n}$ an even-dimensional inner product space with an orthogonal complex structure $J$. Define $\widetilde{R}:=\frac{4}{3}(\Delta-\delta) \widehat{R}_{\mathbb{C} P^{n}}+$ $\frac{1}{3}(4 \delta-\Delta) I_{\Lambda^{2}}$ as a curvature operator on $\Lambda^{2} V$. Then the associated curvature tensor $\widetilde{R}$ on $V$ satisfies:

(i) $\Delta \geq \widetilde{K} \geq \delta, \widetilde{K}(P)=\Delta \Leftrightarrow P$ is J-invariant, $\widetilde{K}(P)=\delta \Leftrightarrow P$ is anti $J$-invariant.

(ii) $\widetilde{R}$ is Einstein: $\widetilde{\mathrm{Ric}}=(\Delta+2 \delta(n-1))\langle$,$\rangle .$

(iii) $\widetilde{R}_{2}$ has $(n-1) \frac{4}{3}(4 \delta-\Delta)$ as an eigenvalue, with eigenvector $J$.

Proof. $\widehat{R}_{\mathbb{C} P^{n}}\left(v_{1} \wedge v_{2}\right):=\frac{1}{4}\left(v_{1} \wedge v_{2}+J v_{1} \wedge J v_{2}+2\left\langle v_{1}, J v_{2}\right\rangle J\right)$, where $\langle J, u \wedge w\rangle=$ $\langle u, J w\rangle$. Thus $\widehat{\widetilde{R}}$ can be written

$$
\widehat{\widetilde{R}}\left(v_{1} \wedge v_{2}\right)=\delta v_{1} \wedge v_{2}+\frac{1}{3}(\Delta-\delta) J v_{1} \wedge J v_{2}+\frac{2}{3}(\Delta-\delta)\left\langle v_{1}, J v_{2}\right\rangle J
$$

If $P$ is a 2-plane with orthonormal basis $v_{1}, v_{2}$, then

$$
\begin{aligned}
\widetilde{K}(P) & =\left\langle\widetilde{R}\left(v_{1}, v_{2}\right) v_{2}, v_{1}\right\rangle=\left\langle\widehat{\widetilde{R}}\left(v_{1} \wedge v_{2}\right), v_{1} \wedge v_{2}\right\rangle \\
& =\delta+(\Delta-\delta)\left\langle v_{1}, J v_{2}\right\rangle^{2}
\end{aligned}
$$

from which (i) follows directly. 
Property (ii) is a direct calculation using $\operatorname{Ric}_{\mathbb{C} P^{n}}=\frac{1}{2}(n+1)\langle\rangle,, \operatorname{Ric}_{I_{\wedge^{2} v^{2 n}}}=$ $(2 n-1)\langle$,$\rangle . To prove (iii), note that from the definitions of \widehat{\widetilde{R}}$ and $\widehat{R}_{2}$ and (2.4), one obtains

$$
\begin{aligned}
\widetilde{R}_{2}\left(v_{1} \wedge v_{2}\right)= & 2(\Delta+\delta(2 n-3)) v_{1} \wedge v_{2}-\frac{2}{3}(\Delta-\delta) J v_{1} \wedge J v_{2} \\
& -\frac{4}{3}(\Delta-\delta)\left\langle v_{1}, J v_{2}\right\rangle J .
\end{aligned}
$$

Now we may choose an orthonormal basis $e_{1}, \ldots, e_{2 n}$ of $V^{2 n}$ so that $J=$ $\sum_{i=1}^{n} e_{2 i-1} \wedge e_{2 i}$, i.e., $J\left(e_{2 i-1}\right)=-e_{2 i}, J\left(e_{2 i}\right)=e_{2 i-1}$, and $J^{2}=-I$. Using this expression for $J$, one now computes directly from (2.20) that $\widetilde{R}_{2} J=$ $\frac{4}{3}(n-1)(4 \delta-\Delta) J$. Q.E.D.

For future reference, we now record the action of $\widehat{\widetilde{R}}$ on the orthonormal basis $\left\{e_{i}\right\}$ such that $J=\sum_{i=1}^{n} e_{2 i-1} \wedge e_{2 i}$. (We assume $j \neq i$.)

$$
\begin{aligned}
& \widehat{\widetilde{R}}\left(e_{2 i-1} \wedge e_{2 i}\right)=\Delta e_{2 i-1} \wedge e_{2 i}+\frac{2}{3}(\Delta-\delta) \sum_{j \neq i} e_{2 j-1} \wedge e_{2 j}, \\
& \widehat{\widetilde{R}}\left(e_{2 i-1} \wedge e_{2 j}\right)=\delta e_{2 i-1} \wedge e_{2 j}-\frac{1}{3}(\Delta-\delta) e_{2 i} \wedge e_{2 j-1}, \\
& \widehat{\widetilde{R}}\left(e_{2 i} \wedge e_{2 j}\right)=\delta e_{2 i} \wedge e_{2 j}+\frac{1}{3}(\Delta-\delta) e_{2 i-1} \wedge e_{2 j-1}, \\
& \widehat{\widetilde{R}}\left(e_{2 i-1} \wedge e_{2 j-1}\right)=\delta e_{2 i-1} \wedge e_{2 j-1}+\frac{1}{3}(\Delta-\delta) e_{2 i} \wedge e_{2 j} .
\end{aligned}
$$

Remark. This action completely determines $\widetilde{R}$ (since the curvature operator determines the curvature tensor) in the sense that if $\widehat{R}$ is any curvature operator on $\wedge^{2} V^{2 n}$ for which there is an orthonormal basis $\left\{e_{i}\right\}$ of $V^{2 n}$ on which $\widehat{R}$ satisfies $(2.21 \mathrm{a})-(2.21 \mathrm{~d})$, then $\widehat{R}=\widehat{\widetilde{R}}$ and consequently $R=\widetilde{R}$, where $J=\sum_{i=1}^{n} e_{2 i-1} \wedge e_{2 i}$.

Our goal now is to prove the following proposition.

Proposition 2.22. Let $R$ be a curvature tensor on $V^{2 n}$ satisfying $\Delta \geq K \geq \delta$ $(\Delta>\delta)$ and $R_{2}$ has $\frac{4}{3}(n-1)(4 \delta-\Delta)$ as an eigenvalue. Then there is an orthogonal complex structure $J$ on $V^{2 n}$ such that $R=\widetilde{R}$.

Propositions 2.17 and 2.22 form Theorem 1.4 of $\S 1$. The strategy for the proof of Proposition 2.22 is as follows:

(2.22i) From the remark after Proposition 2.9, if $X$ is a solution to $R_{2} X=$ $\frac{4}{3}(n-1)(4 \delta-\Delta) X$, then we may assume $X=\sum_{i=1}^{n} e_{2 i-1} \wedge e_{2 i},\left\{e_{i}\right\}$ an orthonormal basis of $V . X$ will serve as the orthogonal complex structure on $V$.

(2.22ii) Use Corollary 2.13 to compute $\left\langle\widehat{R}\left(e_{i} \wedge e_{j}\right), e_{l} \wedge e_{k}\right\rangle=R_{i j k l}$ for all possible $i, j, k, l$. This step consists of a lot of messy linear algebra, and we apologize for its ugliness.

(2.22iii) From (2.22ii), verify that $\widehat{R}$ satisfies $(2.21 \mathrm{a})-(2.21 \mathrm{~d})$, so by the remark after (2.21d), we conclude $R=\widetilde{R}$. 
In order to accomplish (2.22ii), we will need some facts concerning "extremal" components $R_{i j k l}$. In the upcoming proposition, $i, j, k, l, l^{\prime}$ will, unless otherwise stated, stand for different numbers, and subscripts will refer to the fixed orthonormal basis $\left\{e_{i}\right\}$ of $V$ from (2.22i). Also, we shall freely use the symmetries of $R$ from (2.1) and (2.2).

Proposition 2.23. (1) If $K_{i j}$ is extremal (say equal to $\Delta$ or $\delta$ ) then for all $k \neq i, j$ (distinct), $R_{i j k i}=0$.

(2) If $R_{i j k l}$ is extremal (say equal to $\frac{2}{3}(\Delta-\delta)$ ) then for all $l^{\prime} \neq i, j, k, l$, $R_{i j k l^{\prime}}=0$.

(3) If $K_{i j}=K_{i l}=K_{i k}=K_{j k}=K_{j l}=K_{l k}=\delta$ then $R_{i j k l}=0$.

Proof. (1) $K_{i j}=R_{i j j i}=\left\langle R\left(e_{1}, e_{j}\right) e_{j}, e_{i}\right\rangle$ is extremal, so $\forall k \neq i, j$,

$$
0=\left.\frac{d}{d \theta}\right|_{\theta=0}\left\langle R\left(e_{i}, \cos \theta e_{j}+\sin \theta e_{k}\right)\left(\cos \theta e_{j}+\sin \theta e_{k}\right), e_{i}\right\rangle
$$

and this yields (1).

$$
0=\left.\frac{d}{d \theta}\right|_{\theta=0}\left\langle R\left(e_{i}, e_{j}\right) e_{k}, \cos \theta e_{l}+\sin \theta e_{l}^{\prime}\right\rangle .
$$

(3) The assumptions here imply that

$$
K_{j \pm l, i \pm k}=K_{i \pm l, j \pm k}=\delta
$$

so (3) follows from (2.8A) and (2.8B). We shall prove (2.24) for the special case $K_{j+l, i+k}=\delta$; all the other cases are similar. Now

$$
K_{j+l, i}=\left\langle R\left(\frac{e_{j}+e_{l}}{\sqrt{2}}, e_{i}\right) e_{i}, \frac{e_{j}+e_{l}}{\sqrt{2}}\right\rangle=\frac{1}{2}\left\{K_{i j}+K_{i l}+2 R_{i j l i}\right\}
$$

since $K_{i j}=K_{i l}=\delta, R_{i j l i}=0$ from (1), thus $K_{j+l, i}=\delta$. Similarly, $K_{j+l, k}=$ $\delta$. Now $K_{j+l, i}=\delta$ is also extremal, so (1) again yields $R_{j+l, i, k, j+l}=0$. Thus we obtain $K_{j+l, i+k}=\frac{1}{2}\left\{K_{j+l, i}+K_{j+l, k}+2 R_{j+l, i, k, j+l}\right\}=\delta$. As mentioned, all other cases in (2.24) are similar and one uses all six equalities in (3) to accomplish them. Q.E.D.

Proof of Proposition 2.22. Let $X=\sum_{i=1}^{n} e_{2 i-1} \wedge e_{2 i}$ be a solution to $R_{2} X=$ $\frac{4}{3}(n-1)(4 \delta-\Delta) X$ (cf. $\left.(2.22 \mathrm{i})\right)$. We have found it convenient to examine components of $R$ taking the parity into consideration. We note again that different letters will stand for different numbers unless otherwise indicated. Now (2.14) can be written $\forall i \neq j \in\{1, \ldots, n\}$

$$
\delta=K_{2 i-1,2 j-1}=K_{2 i, 2 j}=K_{2 i-1,2 j}=K_{2 i, 2 j-1}
$$

and (2.15), together with Proposition 2.7, yields $\forall i \neq j$,

$$
\begin{aligned}
K_{(2 i-1)+(2 j-1), 2 i+2 j} & =K_{(2 i-1)-(2 j-1), 2 i-2 j}=K_{2 i-(2 j-1),(2 i-1)+2 j} \\
& =K_{2 i+(2 j-1),(2 i-1)-2 j}=\Delta
\end{aligned}
$$


and

$$
\begin{aligned}
K_{(2 i-1)-(2 j-1), 2 i+2 j} & =K_{(2 i-1)+(2 j-1), 2 i-2 j}=K_{2 i+(2 j-1),(2 i-1)+2 j} \\
& =K_{2 i-(2 j-1),(2 i-1)-2 j}=\delta .
\end{aligned}
$$

As one can see from (2.15), (2.25), (2.26i), (2.26ii), and Proposition 2.23, a huge number of components $R_{i j k l}=\left\langle\widehat{R}\left(e_{i} \wedge e_{j}\right), e_{l} \wedge e_{k}\right\rangle$ will equal zero. Before doing the computations for

$$
\widehat{R}\left(e_{i} \wedge e_{j}\right)=\sum_{l<k}\left\langle\widehat{R}\left(e_{i} \wedge e_{j}\right), e_{l} \wedge e_{k}\right\rangle e_{l} \wedge e_{k}=\sum_{l<k} R_{i j k l} e_{l} \wedge e_{k},
$$

we compute the value of two more types of components of $R$.

Proposition 2.27. (i) $\forall i=1, \ldots, n, K_{2 i-1,2 i}=\Delta$.

(ii) $R_{2 i-1,2 j, 2 i, 2 j-1}=R_{2 j, 2 i, 2 i-1,2 j-1}=\frac{1}{3}(\Delta-\delta)$.

Proof. (i) We use the notation $\operatorname{Ric}(x):=\operatorname{Ric}(x, x)$. Compute

$$
\operatorname{Ric}\left(e_{2 i-1}\right)=\sum_{\substack{k \neq i \\ 1 \leq k \leq n}}\left(K_{2 k, 2 i-1}+K_{2 k-1,2 i-1}\right)+K_{2 i, 2 i-1}=2 \delta(n-1)+K_{2 i, 2 i-1}
$$

(from (2.25)). Thus we have

$$
\operatorname{Ric}\left(e_{2 i-1}\right)+R\left(e_{2 j-1}\right)=4 \delta(n-1)+K_{2 i, 2 i-1}+K_{2 j, 2 j-1} .
$$

Also, $\operatorname{Ric}\left(e_{2 i-1}, e_{2 j-1}\right)=\sum_{k=1}^{n}\left(R_{2 k, 2 i-1,2 j-1,2 k}+R_{2 k-1,2 i-1,2 j-1,2 k-1}\right)$. In this last expression, every term equals zero: for $k \neq i, j$,

$$
\begin{array}{ll}
R_{2 k, 2 i-1,2 j-1,2 k}=0 & \text { by } K_{2 k, 2 i-1}=\delta \text { and } 2.23(1), \\
R_{2 k-1,2 i-1,2 j-1,2 k-1}=0 & \text { by } K_{2 k-1,2 i-1}=\delta \text { and } 2.23(1), \\
R_{2 i, 2 i-1,2 j-1,2 i}=0 & \text { by } K_{2 i, 2 j-1}=\delta \text { and } 2.23(1),
\end{array}
$$

and so on. We thus get

$$
\begin{aligned}
4 \delta(n-1)+ & K_{2 i, 2 i-1}+K_{2 j, 2 j-1}=\operatorname{Ric}\left(e_{2 i-1}\right)+\operatorname{Ric}\left(e_{2 j-1}\right) \\
= & \operatorname{Ric}\left(e_{2 i-1}+e_{2 j-1}\right)=2 \operatorname{Ric}\left(\frac{e_{2 i-1}+e_{2 j-1}}{\sqrt{2}}\right) .
\end{aligned}
$$

Now the Ricci form may be computed using any orthonormal basis, and so we compute $\left(\operatorname{Ric}\left(e_{2 i-1}+e_{2 j-1}\right) / \sqrt{2}\right)$ using the orthonormal basis $\left(e_{2 i-1} \pm e_{2 j-1}\right) / \sqrt{2},\left(e_{2 i} \pm e_{2 j}\right) / \sqrt{2}, e_{2 k}, e_{2 k-1}, k \neq i, j$. This yields

$$
\begin{aligned}
\operatorname{Ric}( & \left.\frac{e_{2 i-1}+e_{2 j-1}}{\sqrt{2}}\right)=K_{(2 i-1)+(2 j-1),(2 i-1)-(2 j-1)} \\
& +K_{(2 i-1)+(2 j-1), 2 i+2 j} \\
& +K_{(2 i-1)+(2 j-1), 2 i-2 j} \\
& +\sum_{k \neq i, j}\left(K_{(2 i-1)+(2 j-1), 2 k}+K_{(2 i-1)+(2 j-1), 2 k-1}\right) .
\end{aligned}
$$


$(2.30 \mathrm{a})=K_{2 i-1,2 j-1}=\delta$ from (2.25), $(2.30 \mathrm{~b})=\Delta$ and $(2.30 \mathrm{c})=\delta$ from (2.26i) and (2.26ii), respectively, and each term in $(2.30 \mathrm{~d})=\delta$ from arguments as in Proposition 2.23(3). (2.30) yields

$$
\operatorname{Ric}\left(\frac{e_{2 i-1}+e_{2 j-1}}{\sqrt{2}}\right)=2 \delta(n-1)+\Delta
$$

which, combined with (2.29), yields $K_{2 i, 2 i-1}=K_{2 j, 2 j-1}=\Delta$.

Remark. We now know that relative to the $\left\{e_{i}\right\}$ basis, every $K_{i j}$ is extremal (equal to $\delta$ or $\Delta$ ) so one gets $\forall_{i, j, k}$ (distinct) $R_{i j k i}=0$ from Proposition 2.23(1). This shows that the $\left\{e_{i}\right\}$ diagonalize Ric, and a computation now shows $\operatorname{Ric}\left(e_{2 i}\right)=\operatorname{Ric}\left(e_{2 i-1}\right)=\Delta+2 \delta(n-1)$, so we conclude

$$
\text { Ric }=(\Delta+2 \delta(n-1))\langle,\rangle .
$$

Thus $R$ has the same Ricci tensor as $\widetilde{R}$, and hence the same scalar curvature. If one could show "directly" that the Weyl portion [2, p. 48] $W$ of $R$ equals that of $\widetilde{R}$, then we could conclude that $R=\widetilde{R}$ since the $O(n)$-irreducible pieces agree. Unfortunately, while $\widetilde{W}$ is computable, it is not clear how to compute $W$ a priori.

Proof of 2.27 (ii). We need only show

$$
R_{2 i-1,2 j, 2 i, 2 j-1}=\frac{1}{3}(\Delta-\delta)
$$

since $R_{2 i-1,2 j, 2 i, 2 j-1}=\frac{2}{3}(\Delta-\delta) \quad($ see $(2.15)$ ) and the Bianchi identity (see (2.3)) yield the other equality in 2.27 (ii).

From $(2.8 \mathrm{~A})$ and $(2.8 \mathrm{~B})$, we have

$$
\begin{aligned}
& R_{2 i-1,2 j, 2 i, 2 j-1} \\
& =\frac{1}{6}\left\{K_{(2 i-1)+(2 j-1), 2 j+2 i}+K_{(2 i-1)-(2 j-1), 2 j-2 i}\right. \\
& \quad-K_{(2 i-1)-(2 j-1), 2 j+2 i}-K_{(2 i-1)+(2 j-1), 2 j-2 i}+K_{2 j-(2 j-1),(2 i-1)+2 i} \\
& \left.\quad+K_{2 j+(2 j-1),(2 i-1)-2 i}-K_{2 j+(2 j-1),(2 i-1)+2 i}-K_{2 j-(2 j-1),(2 i-1)-2 i}\right\} \\
& =\frac{1}{3}(\Delta-\delta)+\frac{1}{6}\left\{K_{2 j-(2 j-1),(2 i-1)+2 i}+K_{2 j+(2 j-1),(2 i-1)-2 i}\right. \\
& \left.\quad-K_{2 j+(2 j-1),(2 i-1)+2 i}-K_{2 j-(2 j-1),(2 i-1)-2 i}\right\}
\end{aligned}
$$

(this equality follows from (2.26i) and (2.26ii)). Now arguing as in Proposition 2.23(3) one shows that (all four of) $K_{2 j \pm(2 j-1),(2 i-1) \pm 2 i}=\delta$, and 2.27(ii) follows. Q.E.D.

We now gather up various components of $R$ and, using Proposition 2.23, show that most of them are zero. Our convention that distinct letters mean distinct numbers is now in force, and the indices will vary from 1 to $n$ (there will be some redundancy, but we hope this will be helpful for computing the various components of $\widehat{R}$ ). 


$$
\begin{aligned}
R_{2 i-1,2 j, 2 k, 2 l} & =R_{2 i-1,2 j, 2 k, 2 l-1}=R_{2 i-1,2 j, 2 k-1,2 l-1} \\
& =R_{2 i, 2 j, 2 k, 2 l}=R_{2 i, 2 j, 2 k, 2 l-1} \\
& =R_{2 i, 2 j, 2 k-1,2 l-1}=R_{2 i-1,2 j-1,2 k, 2 l} \\
& =R_{2 i-1,2 j-1,2 k, 2 l-1}=R_{2 i-1,2 j-1,2 k-1,2 l-1}=0 .
\end{aligned}
$$

This follows from Proposition 2.23(3), together with (2.25) (where one can use $l$ or $k$ in place of any of the $i$ 's or $j$ 's).

Remark. (2.33) shows that to compute $\widehat{R}\left(e_{2 i-1} \wedge e_{2 j}\right), \widehat{R}\left(e_{2 i} \wedge e_{2 j}\right)$, and $\widehat{R}\left(e_{2 i-1} \wedge e_{2 j-1}\right)$, we need only compute terms of the form $R_{2 i-1,2 j, a, b}$ where $a$ or $b=2 i, 2 i-1,2 j$, or $2 j-1$, and so on, since all other components in 2.26 are zero.

$$
\begin{aligned}
R_{2 i, 2 j, 2 k, 2 i} & =R_{2 i, 2 j, 2 k-1,2 i}=R_{2 i, 2 j-1,2 k-1,2 i}=R_{2 i-1,2 j, 2 k, 2 i-1} \\
& =R_{2 i-1,2 j, 2 k-1,2 i-1}=R_{2 i-1,2 j-1,2 k-1,2 i-1}=0 .
\end{aligned}
$$

This follows from the remark after (2.31).

$$
\begin{aligned}
R_{2 i, 2 i-1,2 k, 2 j} & =R_{2 i, 2 i-1,2 k-1,2 j}=R_{2 i, 2 i-1,2 k, 2 j-1} \\
& =R_{2 i, 2 i-1,2 k-1,2 j-1}=0 .
\end{aligned}
$$

This follows from (2.15), together with Proposition 2.23(2).

$$
\begin{aligned}
R_{2 i, 2 i-1,2 i, 2 j} & =R_{2 i, 2 i-1,2 i, 2 j-1}=R_{2 i, 2 i-1,2 i-1,2 j} \\
& =R_{2 i, 2 i-1,2 i-1,2 j-1}=0 .
\end{aligned}
$$

Although (2.36) could be derived as (2.34) was, to keep our distinct letter, number convention, we say that this follows from $K_{2 i-1,2 i}=\Delta$, and Proposition 2.23(1). Finally, we mention three more components which equal zero for reasons other than $(2.33)-(2.36)$ :

$$
R_{2 i-1,2 j, 2 k, 2 i}=R_{2 i-1,2 j, 2 k-1,2 i}=R_{2 i-1,2 j, 2 k-1,2 j-1}=0 .
$$

For example, if one "expands" $R_{2 i-1,2 j, 2 k, 2 i}$ into curvature components as in $(2.8 \mathrm{~A})$ and $(2.8 \mathrm{~B})$, one computes that each term in $(2.8 \mathrm{~A})$ equals $\delta$, by arguments similar to Proposition $2.23(3)$, while each term in $(2.8 \mathrm{~B})$ equals $\frac{1}{4}(\Delta+3 \delta)$ by the same method. Thus the terms in $(2.8 \mathrm{~A})$ and $(2.8 \mathrm{~B})$ cancel separately, yielding the first term of (2.37) equal to zero, and the other terms are proved zero similarly.

When computing $\widehat{R}\left(e_{2 i-1} \wedge e_{2 j}\right)$, the terms in (2.37) are the only components whose values are not known on the basis of the previous work. Other components can be shown to be zero by permuting indices. Finally, using (2.15), (2.25), 2.27(i), 2.27(ii), and (2.33)-(2.37) one checks that $\widehat{R}$ has exactly the same output as in (2.21a)-(2.21d) using (2.26). We mention that $(2.21 \mathrm{a})$ is the easiest to verify, but in all cases, one has to compute $\left\langle\widehat{R}\left(e_{2 i-1} \wedge e_{2 i}\right), e_{a} \wedge e_{b}\right\rangle$, $\left\langle\widehat{R}\left(e_{2 i-1} \wedge e_{2 j}\right), e_{a} \wedge e_{b}\right\rangle$, and so on, modulo the previous remarks, for $a, b=2 i$, $2 i-1,2 j, 2 j-1,2 k, 2 k-1,2 l$, or $2 l-1$. Of course, once one has verified the analog for (2.21a) and (2.21b) many of the components in (2.21c) 
and $(2.21 \mathrm{~d})$ follow from the symmetries of $\widehat{R}$. This last horrible calculation completes the proof of Proposition 2.2, which, when combined with Proposition 2.17 , yields Theorem 1.4 of $\S 1$, whose corollary is obtained by taking $\delta=\frac{\Delta}{4}$. Q.E.D.

\section{An application to the Sphere Theorem}

We now prove Theorem 1.3. Fix an orientation on $M$. The assumption $\Delta(p) \geq K(p) \geq \Delta(p) / 4 \geq 0$ yields, by Proposition 2.9, that the Weitzenböck operator, $R_{2}$, is nonnegative at each point, i.e.

$$
\left\langle R_{2} X, X\right\rangle(p) \geq 0 \quad \forall X \in \bigwedge^{2} T_{p} M, \forall p \in M, \text { if } M \text { is even-dimensional . }
$$

In the odd-dimensional case, we get $\left\langle R_{2} X, X\right\rangle(p) \geq \frac{\Delta}{2}(p)|X|^{2}(p)$. Since $M$ is compact and oriented, $b_{2}(M) \neq 0$ implies, by the Hodge-DeRham Theorem, that there is a nonzero harmonic two-form, $X$, on $M$. From (2.5), (2.6), and (3.1), we conclude that $X$ is parallel and satisfies $R_{2} X \equiv 0$. Thus if $M$ is odddimensional, we get $\Delta \equiv 0$, so we may now assume $\operatorname{dim}(M)=2 n$. Normalize $X$ so that $|X|^{2} \equiv n$ ( $X$ has constant length since it is parallel). If $M$ is not flat, then there is a $p \in M$ such that $\Delta(p)>0$. At any such $p$, we have, in the notation of Proposition 2.22, $\Delta(p)>\delta=\Delta(p) / 4$, and a nonzero solution of $R_{2} X_{p}=0$. Thus, at $p$ we can assume (cf. the remark after Proposition 2.9)

$$
X_{p}=\sum_{i=1}^{n} e_{2 i-1} \wedge e_{2 i},
$$

where $\left\{e_{i}\right\}$ forms an orthonormal basis for $T_{p} M$, and invoke Theorem 1.4(ii) (or its corollary) to conclude

$$
R_{p}=\Delta(p) R_{\mathbb{C} P^{n}}
$$

where $R_{p}$ is the curvature tensor of $M$ at $p$, and $J=X_{p}$ to define $R_{\mathbb{C} P^{n}}$. From (3.3) and Proposition 2.17(ii), we conclude that the Ricci tensor of $M$ satisfies

$$
\operatorname{Ric}_{p}=\Delta(p) \cdot \frac{1}{2}(n+1)\langle,\rangle(p) .
$$

Since (3.4) is trivially valid at any point where $\Delta(p)=0$, we conclude that (3.4) is valid on all of $M$, and now a standard argument shows that $\Delta$ must be a constant, and $M$ not flat implies $\Delta$ must be a positive constant. Now (3.2) and (3.3) are valid at every point in $M$, which shows that $X$ yields a parallel orthogonal almost complex structure on $M$. This implies, once $M$ is given the orientation compatible with $X$, that $X$ is integrable and endows $M$ with a Kähler structure $[10 ; 11$, p. 262]. $M$ has constant holomorphic sectional curvature $\Delta$ (by Proposition 2.17(i)) and is simply-connected by Synge's theorem [3] (although other proofs are available once we know $M$ is Kähler and positively curved [8]), hence [9, p. 170] $M$ is biholomorphically isometric 
to $\mathbb{C} P^{n}$ with metric $\frac{1}{\Delta} g_{\text {can }}$, where $g_{\text {can }}$ is the canonical metric on $\mathbb{C} P^{n}$ with constant holomorphic sectional curvature 1. Q.E.D.

As immediate corollaries, we add that if $\Delta: M \rightarrow(0, \infty)$, then $\mathbb{C} P^{n}$ is the only possibility for $M$, and if $\Delta: M \rightarrow(\varepsilon, \infty)$, where $\varepsilon>0$, then one may replace "compact" by "complete" in the hypotheses on $M$, and still conclude that $\mathbb{C} P^{n}$ is the only possibility.

\section{BIBLIOGRAPHY}

1. M. Berger, Sur quelques variétés Riemanniennes suffisamment pincées, Bull. Soc. Math. France 88 (1960), 57-71.

2. A. Besse, Einstein manifolds, Springer-Verlag, Berlin, Heidelberg, and New York, 1987.

3. J. Cheeger and D. Ebin, Comparison theorem in Riemannian geometry, North-Holland, Amsterdam, 1975.

4. D. Gromoll and K. Grove, A generalization of Berger's rigidity theorem for positively curved manifolds, Ann. Sci. Ecole Norm. Sup. 20 (1987), 227-239.

5. K. Grove and K. Shiohama, A generalized sphere theorem, Ann. of Math. (2) 106 (1977), 201-211.

6. S. Helgason, Differential geometry, Lie groups and symmetric spaces, 2nd ed., Academic Press, New York, 1978.

[H] D. Hulin, Pinching and Betti numbers, Ann. Global Anal. Geom. 3 (1985), 85-93.

7. H. Karcher, A short proof of Berger's curvature tensor estimates, Proc. Amer. Math. Soc. 26 (1970), 642-644.

8. S. Kobayashi, On compact Kähler manifolds with positive Ricci tensor, Ann. of Math. (2) 74 (1961), 570-574.

9. S. Kobayashi and K. Nomizu, Foundations of differential geometry, vol. 2, Interscience, New York, 1969.

10. A. Newlander and L. Nirenberg, Complex analytic coordinates in almost complex manifolds, Ann. of Math. (2) 65 (1957), 391-404.

11. W. Poor, Differential geometric structures, McGraw-Hill, New York, 1981.

Department of Mathematics, University of Iowa, Iowa City, Iowa 52242 